\title{
High Performance Liquid Chromatographic Method for Simultaneous Quantitation of Luteolin and Quercitin from Dried Whole Plant Powder of Gmelina Arborea Roxb.And Kalanchoe Pinnata (Lam.)Pers.
}

\author{
Hina Shaikh ${ }^{1}$, Dr. Vidya Dighe ${ }^{2}$ \\ ${ }^{1,2}$ Department of Chemistry, Ramnarain Ruia College, Mumbai, India
}

\begin{abstract}
A simple reversed phase high performance liquid chromatographic (RP-HPLC) method was established for simultaneous quantification of luteolin and quercitin in the two medicinally important plants, Gmelina arborea Roxb. and Kalanchoe pinnata(Lam.) Pers. Linear response was found in the concentration range of $1.0-500 \mu \mathrm{g} / \mathrm{mL}$ for Gmelina arborea Roxb. and $0.5-500 \mu \mathrm{g} / \mathrm{mL}$ for Kalancho epinnata(Lam.) Pers. respectively. The relative standard deviation for inter-day and intra-day precision was found to be $<2 \%$. Precision,specificity and robustness studies were carried out, values less than two (\% R.S.D) were observed. Recovery values from 98.53 to 98.93 indicated that the accuracy of the method was good. The proposed developed method was found to be precise, accurate and reproducible and can be used for routine quality control analysis and for the quantitation of quercitin and luteolin in herbal raw materials as well as in their formulations.
\end{abstract}

Keywords: HPLC; luteolin ; quercetin; Gmelina arborea Roxb. and Kalanchoe pinnata(Lam.) Pers.

\section{Introduction}

Validation of analytical methods is mandatory in implementing a quality control system in any analytical laboratory. It provides an assurance of reliability during normal use and can be referred as a process of providing documented evidence of quality for several herbal and traditional drugs. Separation techniques such as chromatography and electrophoresis have been extensively used for quality control of herbal medicine because of their high efficiency and speed. ${ }^{1}$

The medicinal plants (Rasayana) are the plants whose parts (leaves, seeds, stems, roots, fruit, foliage etc.) extracts, infusion, decoctions, powders have been extensively used in the indian traditional (Ayurveda) system of medicine for the treatment of different diseases of human. ${ }^{2}$

Gmelina arborea Roxb.is a rasayana plant and has immunomodulatory activity. ${ }^{3}$

GmelinaarboreaRoxb.is one of the ingredients of "dashmuladikwath" and "bhrihatpanchamool" of ayurveda, which constitutes a number of ayurvedic preparations used as tonics ${ }^{4}$. According to the Ayurvedic literature, $G$. arborea Roxb.have been reported to be used in case of hallucination, fever, dyspepsia, hyperdipsia, hemorrhoids, gastralgia, anasarca, and in burning sensation. It is bitter, sweet, tonic, laxative, galactogogue, and anthelmintic. ${ }^{5}$

Kalanchoe pinnata (Lam.) Pers. is also reported to have immunomodulatory activity. ${ }^{6}$

Immunomodulatory pretreatment with the plant extract ofKalanchoepinnata (Lam.) Pers.and a component (quercitrin flavonoid) has been shown to effectively protect mice against fatal anaphylactic shock ${ }^{9}$. Kalanchoe pinnata (Lam.) Pers. is a medicinal plant useful for abdominal discomfort, boils, bruises, cholera, cuts, diabetes, diarrhea, dysentery, flatulence, headaches , kidney stones, indigestion, insect bites, scabies, sores, urinary insufficiency and wounds. ${ }^{7}$

Literature survey revealed that HPLC methods have been reported for estimation of luteolin and quercetin from different plant sources such as Beagle Dog ${ }^{8}$, Vegetables, Fruits, and Teas ${ }^{9}$ Tibetan medicine Meconopsis quintuplinervia $^{10}$. However, no HPLC method has been reported for simultaneous quantitation of luteolin and quercitin from methanolic extracts of dried whole plant powder of Gmelina arborea Roxb. and Kalanchoe pinnata(Lam.) Pers.

The present developed RP-HPLC method is advantageous compared to all the methods reported above because it uses a simple isocratic mobile phase comprising of $0.2 \%$ orthophosphoric acid and acetonitrile $(60: 40, \mathrm{v} / \mathrm{v})$ to resolve luteolin and quercitin from other phytochemicals present in both the selected plant materials with significantly lower retention times for luteolin and quercitin than those observed in the reported methods. Thus, the overall time required for analysis is reduced. The developed HPLC method was validated using ICH guidelines ${ }^{11}$. The developed and validated HPLC method is simple and fast as compared to the HPLC methods reported in the literature. 


\section{Materials and Methods}

\subsection{Chemicals}

Acetonitrile (purity- $99.8 \%$ ) and trifluoro acetic acid (purity- $99.0 \%$ ) used in the present research work were of HPLC grade and were procured from Merck, India. Distilled water for HPLC was procured from LiChrosolv Merck, India. The reference standards, luteolin (purity $\geq 98 \%$ ) and quercitin (purity $\geq 95 \%$ ), were purchased from SigmaAldrich Chemie GmbH (Aldrich Division, Steinbeim, Germany).

\subsection{Plant material}

Both the plants Gmelina arborea Roxb.and Kalanchoe Pinnata (Lam.) Pers. were collected from Keshav Shrishti, Maharastra. The plant material of Gmelina arborea Roxb.was authenticated from Agharkar Research Institute, Pune, India (Auth.15-193). Herbarium of Kalanchoe Pinnata (Lam.) Pers. was authenticated from Botanical Survey of India, Pune, India (Certificate No. BSI/WRC/Cert./2014) and collection no.HSQ 01.

Both plant materials were washed with water to remove soil particles, dried in shade, finely powdered and then sieved through BSS mesh size 85 and stored in an airtight container at room temperature $\left(25 \pm 2^{\circ} \mathrm{C}\right)$.

\subsection{Preparation of stock solutions}

Stock solution of $1000 \mu \mathrm{g} / \mathrm{mL}$ of luteolin was prepared by dissolving $50.0 \mathrm{mg}$ of accurately weighed luteolin in $10.0 \mathrm{~mL}$ of methanol in a $50.0 \mathrm{~mL}$ standard volumetric flask. The standard volumetric flask was then sonicated in an ultrasonic bath (Model: TRANS-O-SONIC, Frequency: $50 \mathrm{~Hz}$ ) for 5.0 minutes for complete dissolution of luteolin. The contents were then diluted up to the mark with methanol to obtain a solution of $1000 \mu \mathrm{g} / \mathrm{mL}$ of luteolin.

$5.0 \mathrm{~mL}$ of the above stock solution of luteolin was then transferred to $50.0 \mathrm{~mL}$ volumetric flask and the contents were diluted to $50.0 \mathrm{~mL}$ using methanol to obtain working solution of standard luteolin with concentration $100 \mu \mathrm{g} / \mathrm{mL}$. A similar procedure was followed for preparation of standard stock and working solution of quercitin.

\subsection{Sample Preparation}

About $1.000 \mathrm{~g}$ of dried whole plant powder of Gmelina arborea Roxb.was accurately weighed and transferred to a stoppered conical flask and $10.0 \mathrm{~mL}$ of methanol was then added to it. The flask was then shaken at $50 \mathrm{rpm}$, on a conical flask shaker overnight at room temperature $\left(25^{\circ} \mathrm{C}+\right.$ $\left.2^{\circ} \mathrm{C}\right)$. The contents of the flask were filtered through Whatman No.41 filter paper (E. Merck, Mumbai, India). The filtrate was further used as sample solution for the assay experiment. The sample solution was filtered through $0.45 \mu \mathrm{m}$ filter paper before analysis. The same procedure was followed for preparation of extract of whole plant powder of Kalanchoe Pinnata (Lam.) Pers.

\subsection{Chromatography}

Chromatographic analysis was performed using Shimadzu UFLC Prominence chromatograph, equipped with binary gradient pump (LC-20AD), having auto sampler (SIL-20 AC HT), oven (CTO-20 AC) and PDA detector (SPD-M20A). A reversed-phase Dionex C18 (250mm x 4.6mm, $5 \mu \mathrm{m})$ column was used for chromatographic separation. LC solution software was used for data acquisition. Different compositions of solvents were tried as mobile phase. Finally $0.01 \%$ trifluoroacetic acid in acetonitrile and $0.01 \%$ of trifluoroacetic acid in water in the volume ratio of 45:55.was selected which gave a good resolution between the sample components. The flow rate was maintained at $0.5 \mathrm{~mL} / \mathrm{min}$ and the separated components were detected at $254 \mathrm{~nm}$ (luteolin) and $287 \mathrm{~nm}$ (quercitin).

\section{Method Validation}

\section{Validation of the Method}

ICH harmonized tripartite guidelines were followed for the validation of the developed analytical method ${ }^{11}$.

\subsection{Linearity}

Each standard solution of luteolin and quercitin in the concentration range of $0.1 \mu \mathrm{g} / \mathrm{mL}$ to $500.0 \mu \mathrm{g} / \mathrm{mL}$ were injected in triplicates into the chromatographic system, under optimized chromatographic condition. The peak areas were recorded for each injected concentration of standards. The calibration curves of each of the two standards luteolin and quercitin were obtained by plotting graphs of mean peak areas vs. corresponding concentrations. The results listed in Table 1.

\subsection{Limit of detection (LOD) and Limit of quantitation (LOQ)}

The Limit of Detection (LOD) and Limit of Quantification (LOQ) were determined at signal to noise ratios of 3:1 and 10:1 respectively. The LOD and LOQ values obtained for both the components are listed in Table 1.

\subsection{Precision}

The method was validated in terms of repeatability and intermediate precision. Repeatabilty was carried out in the same laboratory, on the same day, by analyzing sample solution of dried whole plant powder of Gmelina arborea Roxb. and Kalanchoe Pinnata (Lam.) Pers. in triplicate. The intermediate precision was carried out in same laboratory, but on three successive days. The values of \% R.S.D. for both the plants was less than 2 indicating that the method is precise and reproducible.

Table 1

\begin{tabular}{|c|c|c|}
\hline Standards & Luteolin & Quercitin \\
\hline Linear range $(\mu \mathrm{g} / \mathrm{mL})(\mathrm{n}=3)$ & 1.0 to 500.00 & 0.50 to 500.00 \\
\hline Correlation coefficient $(\mathbf{r})$ & 0.999 & 0.999 \\
\hline LOD $(\boldsymbol{\mu g} / \mathbf{m L})$ & 0.3 & 0.16 \\
\hline LOQ $(\boldsymbol{\mu g} / \mathbf{m L})$ & 1.0 & 0.5 \\
\hline
\end{tabular}




\section{International Journal of Science and Research (IJSR) \\ ISSN (Online): 2319-7064}

Index Copernicus Value (2013): 6.14 | Impact Factor (2014): 5.611

\subsection{System suitability}

System suitability experiment was performed by injecting six consecutive injections $(10 \mu \mathrm{g} / \mathrm{mL})$ of each bioactive marker, namely luteolin and quercetin, during the start of the method validation. Values with $\%$ RSD of $\leq 2 \%$ were accepted.

\subsection{Specificity}

The specificity of the proposed HPLC method was ascertained by injecting $10.0 \mu \mathrm{L}$ of blank solution to observe for interference, if any, with the peaks of interest in the chromatogram of the sample solution. It was observed that there is no interference from the blank solution. Methanol was taken as blank solution since standard and sample solutions were prepared in methanol.

The chromatograms were compared by overlay. Good correlation was observed between chromatograms obtained from luteolin and quercitin standards and samples at all $R_{\mathrm{f}}$ positions. The peaks of luteolin and quercitin standard were not masked by any peaks arising due to other components present in the sample solution. Thus, it can be concluded that the developed method is specific.

\subsection{Ruggedness}

Ruggedness of the method was studied by determining the effects of small variations of mobile phase composition $( \pm 2 \%)$, and flow rate $(1.00 \pm 0.05 \mathrm{~mL} / \mathrm{min})$. Effect of these deliberate changes on the response (area) and retention time of QC samples of luteolin and quercitinwas observed during the analysis. The results were expressed in terms of $\%$ mean difference. Values within a difference range of $\pm 5 \%$ were accepted.

The amount of luteolin and quercitin from dried whole plant powders of Gmelina arborea Roxb.and Kalanchoe pinnata (Lam.) Pers. obtained by altered method to that obtained by normal method did not show any significant differences. Thus, it can be concluded that the developed method is robust.

\subsection{Stability of standard luteolin and quercitin}

Standard solution containing the mixture of luteolin and quercitin with concentration of $10.0 \mu \mathrm{g} / \mathrm{mL}$ each, was injected in the chromatographic system, under the specified chromatographic conditions at time intervals of $0,12,24,48$ hours .

The chromatograms were recorded and values of peak areas of standard luteolin and standard quercitin were noted for each injected solution, drawn at regular time intervals.

The values of mean peak area, standard deviation (S.D.) and percent relative standard deviations (\%R.S.D) were calculated for standard luteolin and quercitin. No significant degradation was observed within the given time intervals, thus indicating that standard solutions of luteolin and quercitin of concentrations $10.0 \mu \mathrm{g} / \mathrm{mL}$ each are stable for a period of minimum 48 hours and are thus sufficiently stable to perform the method under normal laboratory conditions.

\subsection{Regression Analysis}

The regression analysis of the calibration data was carried out to determine the relationship between the dependent variable (peak area) and the independent variable (concentration). The regression equation for the above data was found to be

Luteolin: $y=47357 x-9145$

Quercitin: $y=59856 x-58539$

The values of the correlation coefficient, intercept and slope were determined for the graph of mean peak area against corresponding concentration aregiven in Table 2.

Table 2

\begin{tabular}{|c|c|c|}
\hline Standards & Luteolin & Quercitin \\
\hline Slope $(\mathbf{m})$ & 47357 & 59856 \\
\hline Intercept (c) & -9145 & -58539 \\
\hline Correlation coefficient (r) & 0.999 & 0.999 \\
\hline
\end{tabular}

\subsection{Recovery}

Recovery tests were carried out to further investigate the accuracy of the method by adding three different concentration levels of the mixed standard solutions to known amounts of Gmelina arborea Roxb.and Kalanchoe pinnata (Lam.) Pers.. The resultant samples were then extracted and analyzed with the described method. The mean percentage recoveries were calculated using the formula: Recovery $(\%)=[($ amount found - original amount $)$ / amount added] x 100. Values within the range of $85-$ $115 \%$ were accepted. Results obtained are tabulated in Table 3.

\section{Application of validated method for the simultaneous quantitation of luteolin and quercitin from dried whole plant powders of Gmelina arborea Roxb. and Kalanchoe pinnata(Lam.) Pers.}

The developed and validated HPLC method was used for quantitation of luteolin and quercitin from dried whole plant powders of Gmelina arborea Roxb.and Kalanchoe pinnata (Lam.) Pers.

$10 \mu \mathrm{L}$ of the sample solution was injected into the chromatographic system. The identities of peaks of luteolin and quercitinin the samplesolution were confirmed by comparing the chromatogram of the sample (Figure 2 and Figure 3) with that of the standard solution of luteolin and quercitin (Figure 1). Retention times of standards of luteolin and quercitin standards were 4.455 minutes and 6.422 minutesrespectively. The retention times of ofluteolin and quercitin from plant powder of Gmelina arborea Roxb.were found to be 4.453 and 6.411 minutes respectively. The retention times of ofluteolin and quercitin from plant powder of Kalanchoe pinnata (Lam.) Pers. were found to be 4.440 and 6.426 minutes respectively 


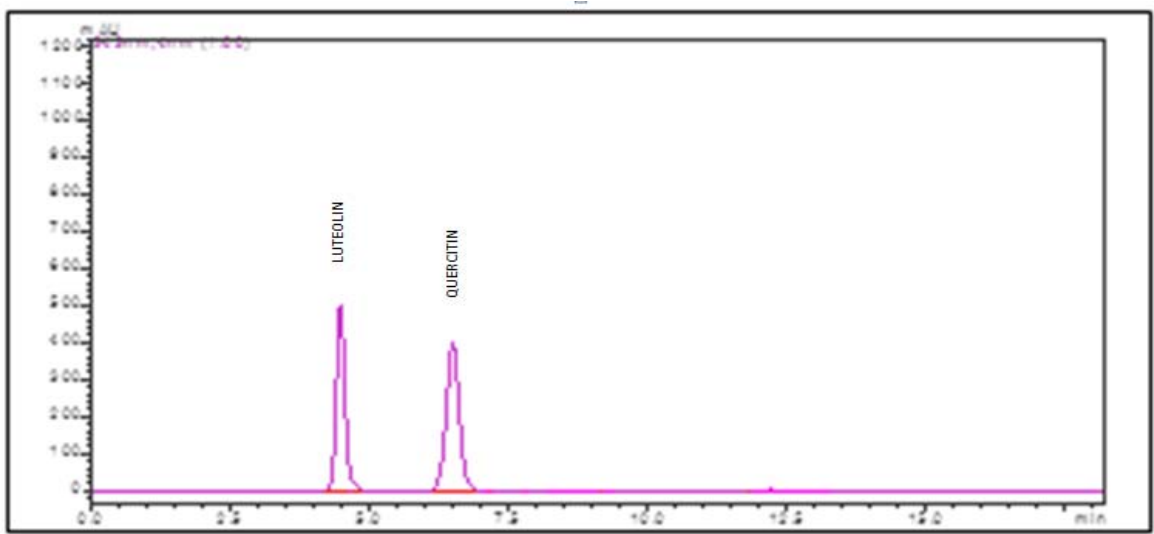

Figure 1: HPLC chromatogram obtained of Standard luteolin and quercitin

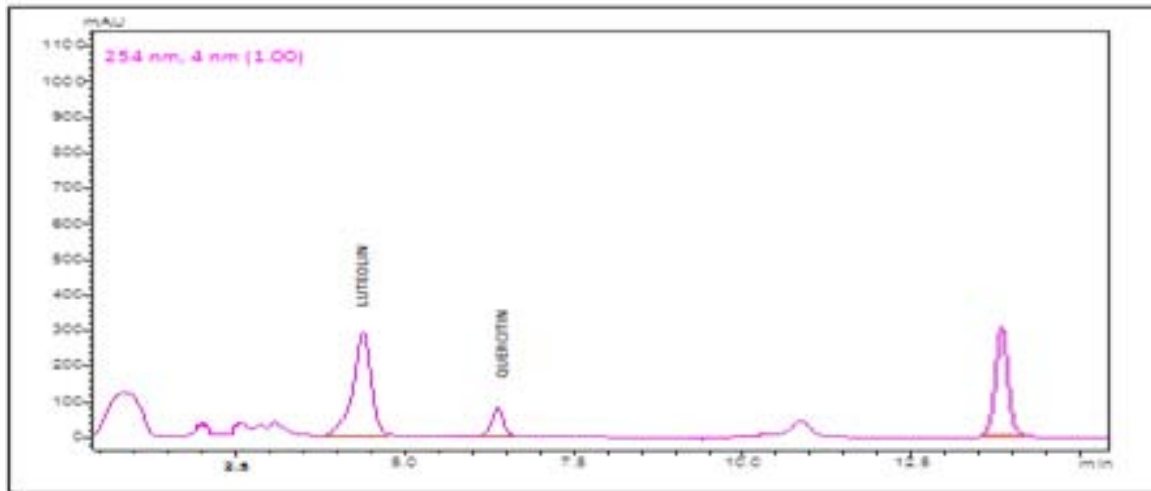

Figure 2: HPLC chromatogram obtained for methanolic extract of dried whole plant powder of Gmelina arborea Roxb.

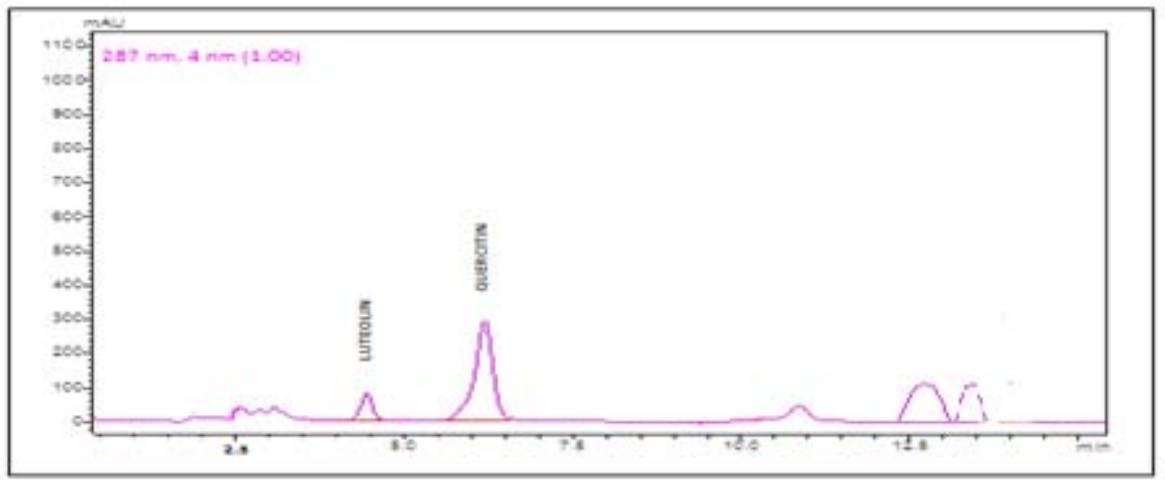

Figure 3: HPLC chromatogram obtained for methanolic extract of Kalanchoe pinnata (Lam.) Pers.

\section{Results}

The method developed to quantify luteolin and quercitin from dried whole plant powders of Gmelina arborea
Roxb.and Kalanchoe pinnata (Lam.) Pers.was validated and the results are tabulated in Table 3

Table 3

\begin{tabular}{|c|c|c|}
\hline \multirow{2}{*}{ Parameters } & \multicolumn{2}{|c|}{ Observations } \\
\hline & Luteolin & Ouercitin \\
\hline \multicolumn{3}{|c|}{ Assay (mg/g) } \\
\hline Plant powderof Gmelina arborea Roxb. & 0.9235 & 0.0836 \\
\hline Plant powder of Kalanchoe pinnata (Lam.) Pers. & 0.0528 & 0.2334 \\
\hline \multicolumn{3}{|c|}{ Percent Recovery $(\%)$} \\
\hline Plant powder of Gmelina arborea Roxb. & 98.53 & 98.86 \\
\hline Plant powder of Kalanchoe pinnata (Lam.) Pers. & 98.65 & 98.93 \\
\hline
\end{tabular}

\section{Conclusion}

A reverse-phase High Performance Liquid Chromatographic method has been developed and validated for simultaneous quantitation of flavone aglycones, quercitin and luteolin from methanolic extracts of dried plant powder of Gmelina arborea Roxb.and Kalanchoe pinnata (Lam.) Pers.. The developed method is simple, precise and accurate and can also be used for routine quality control analysis and for the

\section{Volume 5 Issue 2, February 2016}


quantitation of quercitin and luteolin in herbal raw materials as well as in their formulations.

\section{Conflict of Interest}

Conflict of interest declared none.

\section{References}

[1] Pagliarussi R., Freitas L., Bastos J., A quantitative method for the analysis of xanthine alkaloids in Paulliniacupana (guarana) by capillary column gas chromatography. J. Sep Sci2002; 25:371-4.

[2] Shaikh Arshad ahmed et al. Euphorbia neriifolia Linn. : A phytopharmacological review, IRJP 2 (5) 2011 41-48.

[3] Katiyar C.K., Brindavanam N.B., Tiwari, Narayan D.B.A.: Immunomodulatory products from Ayurveda, current status and future perspectives, Immunomoudlation, S.N.Upadhyay (Ed), Narosa Publishing House, New Delhi,pp163-187.

[4] Gogte VM. Mumbai: The Academic Team of Bharatiya Vidya Bhavan's Swami Prakashananda Ayurveda Research Centre (SPARC); 2000. Ayurvedic Pharmacology and Therapeutic Uses of Medicinal Plants (Dravyagunavignyan).

[5] Sharma PC, Yelne MB, Dennis TJ.. Database on medicinal plants used in Ayurveda. Central Council for Research in Ayurveda and Siddha. Vol. 3. Govt. of India; 2005.

[6] Cruz, E.A., S.A. Da-Silva, M.F. Muzitano, P.M.Silva, S.S. Costa and B. Rossi-Bergmann.Immunomodulatory pretreatment with Kalanchoe pinnata extract and its quercitrin flavonoid effectively protects mice against fatal anaphylactic shock. International Immunopharmacology,2008; 8: 1616-1621.

[7] Samim Sofika and Rajib Jogoi.Herbal receipe prepared during Bohag or Rongali Bihu in Assam , Indian Journal of Traditional Knowledge, 2007;6(3): 417-422.

[8] J. Wu, H. Xing, D. Tang, Y. Gao, X. Yin, Q. Du, X. Jiang and D. Yang. Simultaneous Determination of Nine Flavonoids in Beagle Dog by HPLC with DAD and Application of Ginkgo biloba Extracts on the Pharmacokinetic. Acta Chromatographica 24(2012)4, 627-642

[9] Hiroyuki Sakakibara, Yoshinori Honda, Satoshi Nakagawa, Hitoshi Ashida and Kazuki Kanazawa. Simultaneous Determination of All Polyphenols in Vegetables, Fruits, and Teas. 2002, Rokkodai, Nada-ku, Kobe 657-8501, Japan

[10]Pengpeng Yue, Jing Sun, Changxian Zhang, Runrong Ye, Xuefeng Lu, Yubi Zhou, Shibing Yang and Min Peng HPLC-DAD separation and determination of major active constituents in an important Tibetan medicine Meconopsis quintuplinervia from different regions of Qinghai-Tibet Plateau. Journal of Medicinal Plants Research Vol. 4(11), pp. 1053-1058, 4 June, 2010

[11]ICH Harmonised Tripartite Guideline, Validation of Analytical Procedures: Text and Methodology Nov. 2005; Q2 (R1). 